\title{
ON THE TANGENT BUNDLE OF A NORDEN MANIFOLD ENDOWED WITH A WEYL CONNECTION
}

\author{
RABIA CAKAN AKPINAR
}

$\begin{array}{lll}\text { Date of Receiving } & : & 23.12 .2019 \\ \text { Date of Revision } & : & 13.03 .2020 \\ \text { Date of Acceptance } & : & 18.05 .2020\end{array}$

\begin{abstract}
The aim of this paper is to study the tangent bundle of Norden manifolds endowed with the complete lift of a Weyl connetion. Firstly, the condition for an almost Norden manifold of being a Norden manifold endowed with the Weyl connection is investigated. Finally, the condition for the tangent bundle of an almost Norden manifold of being a Norden manifold endowed with the Weyl connection is investigated.
\end{abstract}

\section{Introduction}

The tangent bundle of a differentiable manifold has an important role for providing many new problems in the study of modern differential geometry. Some studies about metric and connection have been built on the tangent bundle of a differentiable manifold by Yano and Davies [23]. Some extension of metric and connection have been studied on tangent bundle by many authors $[1,4,5,8,14]$.

H. Weyl introduced unified field theory to study a generalization of Riemannian geometry in 1918 [22]. Weyl's theory provided mathematicians to study a new topic. There are many studies devoted to Weyl structure described as a conformal class of metrics preserved by Weyl connection. Folland showed that a Weyl structure is equivalent to a connection on a certain bundle and derived a characterization of Weyl structures in terms of their induced linear connection [6]. Hall has made a study of Weyl connection and their associated holonomy groups [9]. Bejan and Gül have constructed a Weyl structure on tangent bundle of Weyl manifold by using the Sasaki metric [2] Tarafdar and Kundu have studied different types of geometric properties on almost Hermitian manifolds admitting Einstein-Weyl connection [21].

Since 1960, one of the fields in differential geometry that has developed rapidly is the theory of Norden manifolds. Norden investigated the B-metric with respect to almost complex structure on four-dimensional pseudo-Rimannian neutral space [13]. In some studies, different names (anti-Hermitian, pure, Norden) are used for B-metric

2010 Mathematics Subject Classification. 53C07, 55R10.

Key words and phrases. Weyl connection, tangent bundle, complete lift, Norden manifold. Communicated by. Simona Druta-Romaniuc 